Mots. Les langages du politique

\title{
Laurence Rosier, Petit traité de l'insulte
}

Paul Bacot

\section{CpenEdition}

\section{Journals}

Édition électronique

URL : https://journals.openedition.org/mots/1084

DOI : $10.4000 /$ mots. 1084

ISSN : 1960-6001

\section{Éditeur}

ENS Éditions

\section{Édition imprimée}

Date de publication : 1 juillet 2007

Pagination : 116-119

ISBN : 978-2-84788-112-7

ISSN : 0243-6450

\section{Référence électronique}

Paul Bacot, «Laurence Rosier, Petit traité de l'insulte », Mots. Les langages du politique [En ligne], 84 | 2007, mis en ligne le 01 juillet 2009, consulté le 22 avril 2022. URL : http://journals.openedition.org/ mots/1084; DOl : https://doi.org/10.4000/mots.1084 


\section{Petit traité de l'insulte}

Laurence Rosier

2006, Loverval (Belgique), Labor (Liberté, j'écris ton nom), 112 p.

L'insulte en politique se fait rare, mais n'a pas disparu. Qu'on pense à ce président du conseil italien traitant publiquement, il y a quelques années, un eurodéputé allemand de «kapo nazi» et, plus récemment, les électeurs du camp opposé au sien de «coglioni»; ou à ce député français dénonçant une mainmise des homosexuels sur le pouvoir politique en fustigeant les «khmers roses »; ou encore à ce ministre de l'Intérieur reprenant à son compte la stigmatisation de certains habitants du quartier qu'il visitait en parlant d'eux comme de la «racaille»; sans oublier ce maire et président de région lançant «Vous êtes des sous-hommes! » à une délégation de harkis. À ces quelques exemples récents rappelés par Laurence Rosier, on pourrait en ajouter de plus anciens: Simone Veil qualifiée de «tricoteuse de Giscard», ou Robert Schuman accueilli dans l'hémicycle du Palais-Bourbon au cri de «Tiens, v'là l'Boche!».

Entendue comme proférée par des politiciens et/ou dans une enceinte réputée politique, l'insulte politique se fait rare. Le même processus de pacification de la vie politique qui conduisit à remplacer la violence physique par une violence verbale amène aujourd'hui à disqualifier cette dernière. Proférée en face à face et en public, l'insulte fait l'objet de suites pénales, sans que le contexte politicien de son énonciation constitue en quoi que ce soit une excuse. Mais Internet, comme les vestiaires des stades ou les cours de récréation, fournit de larges espaces à la pratique de l'insulte - ce dont témoigne le très riche corpus de l'ouvrage. L'insulte est le produit d'une compétence largement partagée...

L'intérêt de ce petit Petit traité est pluriel. Mais d'un point de vue politologique, il a le grand mérite de montrer que l'insulte peut être politique hors de tout contexte politicien, voire qu'elle l'est toujours, en quelque sorte par nature. Le constat n'est pas formulé en ces termes par l'auteur, qui affirme que «toute injure est d'une certaine façon une injure raciste» (formulation empruntée à Évelyne Larguèche), ou encore que «l'insulte est par essence une haine raciale» (p. 6 et 65 ). Nous dirons quant à nous qu'elle est toujours politisante, au sens où le locuteur fait usage d'un clivage dessinant deux camps opposés, celui dans lequel il s'autopositionne et celui dans lequel il classe le destinataire de l'insulte, censé soit appartenir à cette catégorie stigmatisée, soit ressembler à ceux qui la peuplent.

Mais qu'est-ce qu'une insulte ? La linguiste de l'université libre de Bruxelles, par ailleurs spécialiste reconnue de l'analyse du discours rapporté, synthétise les résultats de ses travaux antérieurs et de ceux d'autres chercheurs (Philippe Ernotte, Dominique Lagorgette, Évelyne Larguèche, Nicolas Ruwet). Si insultare, en latin, c'était «sauter dessus», insulter rend bien toujours compte 
d'une agression - verbale, à côté d'autres formes d'injures et d'outrages: certains gestes, certaines mimiques, certaines attitudes, un certain silence ou une indifférence méprisante. Le caractère "performatif» (selon la terminologie de John Austin) de l'insulte, «forme typiquement linguistique de l'injure», est en effet établi, puisque par le seul fait qu'elle est prononcée, elle provoque, blesse et, parfois, tue. Désignation de l'interlocuteur par le locuteur, l'insulte peut être analysée d'un point de vue pragmatique comme un appellatif en termes d'adresse. Pour insulter, l'insultant peut user du lexique existant, mais aussi faire œuvre de néologie : Laurence Rosier rappelle que le président d'un parti français traita naguère de "fédérastes» les partisans d'une Europe fédérale, après avoir, plusieurs années auparavant, qualifié de «sidaïques » les malades du sida. On pourrait aussi renvoyer à la fabrication prolixe de mots-valises par les anarchistes de la Belle époque.

L'auteur insiste à juste titre sur le fait qu'un mot classé en langue comme insultant peut être utilisé d'une manière non insultante (hypocoristique ou spirituelle, par exemple), et qu'à l'inverse, un mot classé en langue comme non insultant peut être utilisé d'une manière insultante - par exemple dans le cas de ce que Laurence Rosier appelle «l'effet Arletty », en référence à un célèbre quiproquo autour du mot atmosphère. L'énoncé insultant est donc dialogique (référence est faite à Mikhaïl Bakhtine), il est produit dans l'interaction, il joue de la mémoire du mot, c'est-à-dire des sens que celui-ci a acquis au fil de ses emplois. "L'insulte n'est pas un mot de la langue, mais un mot du discours" (p. 87). Dès lors, le même mot a ou n'a pas de portée insultante selon le lieu et les conditions de son énoncé, et son sens ne sera pas nécessairement le même pour celui qui l'emploie, pour celui auquel il est destiné et pour un éventuel tiers-écoutant: «On peut rire de tout, mais pas avec tout le monde», dit-on. L'expression «mettre les rieurs de son côté » prend ainsi tout son sens : il s'agit d'insulter la cible par un discours qui ne sera pas perçu par le public comme insultant, mais seulement comme comique. Et pour nous, cette expression montre bien en quoi la stratégie de l'insulte possède les propriétés de toute stratégie politisante : réduire le camp de l'adversaire et accroître le sien, par le choix d'un clivage et une certaine présentation de celui-ci.

Revenons donc à ce que Laurence Rosier nous dit de cette pratique qu'elle rapproche fort justement du discours raciste. Pour elle, en effet, toute insulte est fondée sur une «représentation stéréotypique » et révèle la manière dont on "pense la différence» (p. 6). Elle « est souvent, presque toujours, le vecteur de la discrimination, du racisme, du sexisme» (p. 17), fonctionnant comme une «prédication par une enclosure classifiante» (p. 67). La forme construite sur le modèle [espèce de + nom de catégorie] est de ce point de vue particulièrement éclairante - que la catégorie instrumentalisée par l'insultant soit animalière,

1. Voir les travaux de Maurice Tournier. 
végétale, sexuelle, physique ou d'une autre sorte. L'insulte porte une représentation stéréotypée et stigmatisante de cette catégorie, identifie l'insulté comme l'un de ses éléments, et par là-même lui assigne les traits identitaires qui sont réputés être les caractéristiques du groupe désigné. Elle «classe l'insulté par stigmatisation» (p.69). L'insulte fonctionne donc toujours plus ou moins comme une métonymie, ou comme une antonomase - ce qui selon nous, dans ce cas, revient à peu près au même. En somme, «l'insulte classe l'insulté par stigmatisation» (p.69). Mais l'auteur ajoute fort justement que, ce faisant, «l'insultant s'engage », se classe lui-même - en opposition, bien sûr!

Pourtant, l'insulté peut refuser, sinon l'identité qui lui est assignée, du moins le caractère insultant de celle-ci. C'est le sens de l'initiative, rappelée dans l'ouvrage, des signataires du fameux «Manifeste des 343 salopes» qui, en 1971, s'appropriaient la façon dont les adversaires de l'interruption volontaire de grossesse dénommaient les femmes qui y avaient recours; ou encore de celle de ces militants italiens arborant des tee-shirts portant l'inscription "Sono un coglione!", en réponse à l'agression verbale de leur principal adversaire. Car dans cette négociation des identités, «c'est la réception comme une insulte qui fait l'insulte». II arrive même que l'insultant soit l'humilié quand l'insulté ne se laisse pas humilier.

Nous avons donc trouvé dans ce passionnant Petittraité une forte illustration du processus général de politisation, entendu comme la mise en scène de deux camps opposés de part et d'autre d'un clivage à la portée cognitive générale, susceptible d'expliquer les situations conflictuelles les plus variées, par élargissement de la conflictualité. Traiter quelqu'un de «pédé», par exemple, c'est bien considérer que l'opposition entre hétérosexuels et homosexuels permet de rendre compte de conjonctures conflictuelles multiples - dans la pratique automobile, sportive ou littéraire, voire dans la vie politicienne. Que l'adversaire soit effectivement considéré comme homosexuel n'a dès lors qu'un intérêt secondaire : il est classé parmi les «pédés», ce qui est censé permettre tout à la fois d'expliquer, voire d'anticiper son comportement et de rattacher l'incident ou le conflit présent à toutes une série d'incidents ou de conflits passés, présents et à venir, ici et ailleurs, présentés comme actualisant la même logique conflictuelle.

Reste à pointer ce qui fait la spécificité de cette forme particulière de politisation qu'est l'insulte. Ne serait-ce pas que le clivage qu'elle mobilise se ramène toujours plus ou moins à l'opposition du digne et de l'indigne? Plus précisément, l'indignité censée caractériser l'insulté semble toujours être présentée comme ontologique, essentielle ${ }^{2}$, et donc irrémédiable. En cela, l'insulte est bien toujours «raciste», au sens large. Elle donne à voir une représentation politisante du monde fondée sur une opposition binaire entre dignes

2. L'auteur de l'ouvrage considère qu'une insulte peut n'être que « passagère », p. 55. 
et indignes, que ces derniers soient stigmatisés comme «pédés» ou comme «cons», comme "sales nègres» ou "sales juifs», comme "paysans» ou... - mais laissons au lecteur le plaisir de découvrir dans le Petit traité de Laurence Rosier la richesse infinie de la production linguistique injurieuse!

Paul Bacot

Institut d'études politiques de Lyon, CNRS (Triangle)

paul.bacot@univ-lyon2.fr

\section{Le débat public en apprentissage : aménagement et environnement. Regards croisés sur les expériences françaises et québécoises}

Louis Simard, Laurent Lepage, Jean-Michel Fourniau, Michel Gariépy, Mario Gauthier éd. 2006, Paris, L'Harmattan (Villes et entreprises), 315 p.

Dans un contexte valorisant la démocratie participative sous toutes ses formes, il est impossible d'étudier les conflits autour des questions d'environnement ou d'aménagement sans prendre en considération les canaux qui recueillent la voix des protestataires. Le mouvement écologiste a émergé, à la fin des années soixante, dans les pays industrialisés, en réaction à des projets d'aménagement de grande envergure (Grand Canyon aux États-Unis, par exemple). Les animateurs de ces mobilisations réclamaient davantage de transparence dans la décision et une prise en compte des intérêts des habitants, autant matériels (indemnisation) que d'ordre environnemental (pollution, perte d'un cadre de vie). Face à cette montée des «mécontentements» qui se diffusaient parmi des citoyens ordinaires, les pouvoirs publics ont imaginé des dispositifs permettant d'expliciter la décision prise et de démontrer l'intérêt de ces aménagements. Aux États-Unis et au Québec, ces dispositifs de concertation ont été mis en place dès les années soixante-dix dans le cadre d'une culture associative et politique de négociation entre groupes d'intérêts. En France, le cheminement a été plus lent, plus chaotique aussi avec la procédure d'enquête publique.

L'ouvrage dirigé par Louis Simard, Laurent Lepage, Jean-Michel Fourniau, Michel Gariepy et Mario Gauthier rassemble des textes écrits par des universitaires, des chercheurs mais aussi par des praticiens, textes relatifs aux expériences française et québécoise de débat public en matière d'aménagement et d'environnement. Cette comparaison met en lumière des différences qui sont liées au contexte social, aux cultures de revendication ou aux attitudes à l'égard de la parole contestataire. Deux expériences qui se caractérisent aussi par une longévité différente: le Bureau d'audiences publiques sur l'environnement (BAPE) est né dès les années soixante-dix, tandis que la Commission nationale du débat public (CNDP) n'a qu'une décennie : elle a suivi la loi Barnier du 